\title{
Early but No Long-term Benefit of Regional Compared with General Anesthesia for Ambulatory Hand Surgery
}

Colin J. L. McCartney, M.B.Ch.B., F.C.A.R.C.S.I., F.R.C.P.C., ${ }^{\star}$ Richard Brull, M.D., F.R.C.P.C.† Vincent W. S. Chan, M.D., F.R.C.P.C., $\ddagger$ Joel Katz, Ph.D.,§ Sherif Abbas, M.D.,\| Brent Graham, M.D., F.R.C.S.C.,\# Hugo Nova, M.D., \|

Regan Rawson, R.N., ** Dimitri J. Anastakis, M.D., F.R.C.S.C., F.A.C.S., †† Herbert von Schroeder, M.D., F.R.C.S.C.\#

\begin{abstract}
Background: The purpose of this study was to determine whether either regional anesthesia (RA) or general anesthesia (GA) provided the best analgesia with the fewest adverse effects up to 2 weeks after ambulatory hand surgery.

Methods: Patients undergoing ambulatory hand surgery were randomly assigned to RA (axillary brachial plexus block; $\mathbf{n}=$ $50)$ or $G A(n=50)$. Before surgery, all patients rated their hand pain (visual analog scale) and pain-related disability (Pain-Disability Index). After surgery, eligibility for bypassing the postanesthesia care unit ("fast track") was determined, and pain, adverse effects, and home-readiness scores were measured. On postoperative days 1,7 , and 14, patients documented their pain, opioid consumption, adverse effects, Pain-Disability Index, and satisfaction.
\end{abstract}

Results: More RA patients were fast-track eligible $(P<0.001)$, whereas duration of stay in the postanesthesia care unit was shorter in the RA group $(P<0.001)$. Time to first analgesic request was longer in the $\mathrm{RA}$ group $(P<0.001)$, and opioid consumption was reduced before discharge $(P<0.001)$. In the RA group, the pain ratings measured at $30,60,90$, and $120 \mathrm{~min}$ after surgery were lower $(P<0.001)$, and patients spent less time in the hospital after surgery $(P<\mathbf{0 . 0 0 1})$. More GA patients experienced nausea/vomiting during recovery in the hospital $(P<0.05)$. However, on postoperative days 1,7 , and 14, there were no differences in pain, opioid consumption, adverse effects, Pain-Disability Index, or satisfaction.

Conclusions: Despite significant reduction in pain before discharge from the hospital after ambulatory hand surgery, singleshot axillary brachial plexus block does not reduce pain at home on postoperative day 1 or up to 14 days after surgery when compared with GA. However, RA does provide other significant early benefits, including reduction in nausea and faster discharge from the hospital.
BOTH regional anesthesia (RA) and general anesthesia (GA) are commonly performed for ambulatory hand surgery. Existing nonrandomized prospective and retrospective data show that RA offers several advantages compared with GA during recovery in the hospital after ambulatory hand surgery, including a reduction in opioid and antiemetic consumption, shortened duration of stay in the postanesthesia care unit (PACU) and day surgery unit (DSU), and expedited discharge from the hospital. ${ }^{1}$ Compared with GA for ambulatory surgery of the upper limb, RA is associated with reduced surgical ${ }^{2}$ and nonsurgical ${ }^{3}$ intraoperative times, fewer unanticipated postoperative hospital admissions, ${ }^{2,4}$ and greater patient satisfaction. ${ }^{2}$ In addition, the complete sensory blockade produced by RA techniques may reduce central sensitization and may have a preventive analgesic benefit. $^{5,6}$

However, modern general anesthetic and analgesic techniques have several advantages compared with older agents and may negate any advantage of RA. Newer short-acting general anesthetic agents produce significantly fewer adverse effects, shorter recovery time, ${ }^{7}$ reduced hospital costs, and improved patient satisfaction $^{8}$ compared with older agents. Anesthesiologists are more familiar with providing GA compared with RA, and it follows that GA is the most widely used anesthetic technique for ambulatory surgery. ${ }^{10}$

Although RA has been associated with improved outcomes for patients undergoing hand surgery while they are in the hospital, less is known about what happens after discharge. Cooperation with rehabilitation is important for functional success after surgery, ${ }^{11}$ and good early pain control may facilitate this process. ${ }^{12}$

No previous prospective randomized trials exist to determine whether RA or GA is superior for patients undergoing ambulatory surgery of the upper extremity. Our hypothesis was that RA, by providing good early pain control, would provide patients with better pain control and less pain-related disability at 14 days after surgery.

\section{Materials and Methods}

After approval by the University Health Network Research Ethics Board (Toronto, Canada) and written informed consent, we recruited patients with American Society of Anesthesiologists grade I-III who were undergoing ambulatory hand surgery to this prospective, ran- 
domized, open-label study. Inclusion criteria included age $18-80 \mathrm{yr}$, weight $40-100 \mathrm{~kg}$, and surgery duration greater than $30 \mathrm{~min}$ and less then $3 \mathrm{~h}$. Exclusion criteria were language barrier, contraindication to RA, intolerance to nonsteroidal antiinflammatory drugs, asthma, bleeding diathesis, long-term opioid use, psychiatric history, and pregnancy.

Each patient's medical history and preoperative daily medication consumption were recorded, and all patients rated the intensity of pain in their hand (operative side) using a 100-mm visual analog scale (VAS). In addition, all patients completed the Pain-Disability Index (PDI) ${ }^{13}$ as a preoperative measure of disability due to pain in their hand (operative side). The PDI is a valid self-reported instrument that determines the extent to which pain interferes with normal role functioning in seven daily activities: family/home responsibilities, recreation, social activity, occupation, sexual behavior, self-care, and lifesupport activity.

Using a computer-generated randomization table, patients were allocated to receive either RA or GA immediately before surgery. Patients allocated to RA were taken to the regional block room for peripheral nerve blockade and subsequently transferred to the operating room (OR), whereas those allocated to GA were taken directly to the OR. Standard monitors were applied to all patients, including noninvasive blood pressure monitor, electrocardiograph, and pulse oximeter, and intravenous access was secured on the nonoperative side for commencement of a $0.9 \%$ saline infusion. All patients received intravenous midazolam, $0.03 \mathrm{mg} / \mathrm{kg}$, and ketorolac, $15 \mathrm{mg}$, before induction of anesthesia.

Patients randomized to RA received a transarterial axillary brachial plexus block performed by a skilled anesthesiologist or directly supervised delegate. On the operative side, the shoulder was abducted, and the elbow was flexed to $90^{\circ}$. The axilla was prepared using an aseptic technique, and the axillary artery was palpated. A subcutaneous injection of $2 \%$ lidocaine, $1 \mathrm{ml}$, provided anesthesia to skin. A 1-in, 23-gauge, noninsulated, longbeveled needle (PrecisionGlide; Becton Dickinson, Franklin Lakes, NJ) was then introduced, and on confirmation of needle tip placement immediately posterior to the axillary artery (by negative aspiration of blood), 1.5\% lidocaine, $10 \mathrm{mg} / \mathrm{kg}$, with 1:200,000 epinephrine was injected incrementally. The time at which the anesthesiologist attended the patient, the needle-skin puncture, the time at injection, and the time of onset of surgical anesthesia were all recorded. In addition, the number of needle-skin punctures (i.e., attempts), duration of needle-skin penetration, incidence of transient paresthesiae on RA administration, and any other complications, including pain on injection of the local anesthetic, were noted. Supplementary peripheral nerve blocks at the elbow or wrist were not performed.

Surgical anesthesia at the operative site was deter- mined immediately before incision. Inadequate anesthesia was defined as pain on pinch in the surgical site using Allis forceps. In the event of inadequate anesthesia, a standardized algorithm was followed (standard of practice in our institution): The surgeon first infiltrated the surgical site with $1-2 \%$ lidocaine or $0.25-0.5 \%$ bupivacaine. Fentanyl, $25 \mu \mathrm{g}$, could also be administered every $5 \mathrm{~min}$ up to a maximum of $100 \mu \mathrm{g} / \mathrm{h}$. Patients who had inadequate analgesia despite these measures switched to GA. Inadequate anxiolysis was treated by administration of 10-20 $\mathrm{mg}$ propofol every $5 \mathrm{~min}$ as required.

The quality of the RA was recorded as follows: (1) adequate: no supplemental analgesic or sedation required for surgery to proceed within a 30 -min period from institution of axillary block; (2) inadequate: supplemental analgesic, sedation, or both required for surgery; or (3) failed: GA required for surgery. Patients in the RA group who required GA because of inadequate analgesia, anxiolysis, or both were assessed in the final analysis as part of the RA group (intention-to-treat analysis).

General anesthesia comprised a standardized intravenous regimen including $2-3 \mathrm{mg} / \mathrm{kg}$ propofol and $2 \mu \mathrm{g} / \mathrm{kg}$ fentanyl. A laryngeal mask airway or endotracheal tube was used for airway management during surgery. Patients who required tracheal intubation were paralyzed with $0.6 \mathrm{mg} / \mathrm{kg}$ rocuronium. Maintenance of GA was provided with a 50:50 mixture of oxygen and nitrous oxide and 2-6\% desflurane. Muscle relaxation was antagonized with $50 \mu \mathrm{g} / \mathrm{kg}$ neostigmine and $5-10 \mu \mathrm{g} / \mathrm{kg}$ glycopyrrolate if necessary.

All patients had an upper arm tourniquet applied and inflated to $100 \mathrm{mmHg}$ above their systolic blood pressure (minimum $200 \mathrm{mmHg}$ ). Both the tourniquet pressure and the duration of tourniquet inflation were recorded.

During postoperative recovery in the hospital, pain (VAS pain score of $\geq 40 \mathrm{~mm}$ or patient request for analgesic) was treated with $25-\mu \mathrm{g}$ fentanyl increments every $5 \mathrm{~min}$. When oral fluid intake was initiated, patients received one of two oral analgesic preparations as needed: Tylenol \#3 (300 mg acetaminophen-30 mg codeine-15 mg caffeine per tablet; McNeil Consumer Healthcare, Guelph, Ontario, Canada) or $325 \mathrm{mg}$ acetaminophen-5 mg oxycodone $\mathrm{HCl}$ per tablet if intolerant to codeine.

At the time of discharge from the hospital, patients received a further prescription for Tylenol \#3 or $325 \mathrm{mg}$ acetaminophen-5 $\mathrm{mg}$ oxycodone $\mathrm{HCl}$ and were instructed to take 1-2 tablets every $4 \mathrm{~h}$ as required for pain up to a maximum of 12 tablets/day.

Intraoperatively, the anesthesia induction time, total surgical time, total tourniquet time, and total anesthesia time were recorded. On termination of surgery and anesthesia in the OR, fast-track eligibility using the Modified Aldrete score ${ }^{14}$ was determined. The Modified Aldrete score rates patient activity, respiration, circulation, 
consciousness, and oxygen saturation as a measure of discharge readiness from the PACU to the DSU but has recently been used as a surrogate measure of eligibility to bypass the PACU, i.e., "fast tracking." 15 At 30-min intervals postoperatively, VAS pain scores and Post-Anesthesia Discharge Scoring System home-readiness scores were collected by an unblinded research assistant.

The Post-Anesthesia Discharge Scoring System ${ }^{16}$ is a valid and reliable cumulative index designed to assess eligibility for discharge home after surgery and anesthesia. Durations of stay in the PACU (PACU recovery time) and DSU (DSU recovery time) were also recorded.

At the time of discharge from hospital, patients were given a home diary to complete and return by mail. On postoperative days (PODs) 1, 7, and 14, patients were instructed to document their VAS pain score, daily and cumulative oral analgesic consumption since discharge from the hospital, incidence of nausea or vomiting, incidence of weakness in the operative arm, incidence of paresthesiae (numbness or tingling) in the operative arm, and current VAS satisfaction with anesthetic care. Finally, patients were also instructed to repeat the PDI on POD 14. All patients received telephone calls on days 1,7 , and 14 to remind them to complete and return the diary.

The doses of oral codeine or oxycodone consumed by each patient were converted into equianalgesic doses of oral morphine sulfate to facilitate comparison between both groups. Equianalgesic conversion ratios were used according to the general monograph for opioids in the Canadian Pharmacists Association Compendium of Pharmaceuticals and Specialties ${ }^{17}$ as follows: oral oxycodone: oral morphine sulfate $=1: 2$, and oral codeine:oral morphine sulfate $=3.3: 1$.

\section{Statistics}

Data were analyzed by intention-to-treat using SPSS ${ }^{\circledR}$ version 10.0 software (SPSS Inc., Chicago, IL). Normally distributed continuous data were analyzed using the unpaired Student $t$ test. Nonnormally distributed data were analyzed using the Mann-Whitney $U$ test. Differences in proportions were compared by chi-square test. All repeated variables were corrected using the Bonferroni method. Significance was considered at $P<0.05$.

\section{Sample Size Estimation}

We defined our primary outcome as pain intensity (measured by VAS) on POD 14 after ambulatory hand surgery. Because there were no previous data regarding pain intensity 2 weeks after hand surgery, we calculated a sample size of 100 patients (50 per group) using the Cohen $d$ and a medium effect size correlation (SPSS ${ }^{\circledR}$ SamplePower, version 1.0) with a type I error rate of 0.05 and power of 0.80 . A sample size of 100 patients also ensured adequate power to detect a difference in pain intensity between the RA and GA groups on POD 1.
This was based on data from a previous randomized trial comparing interscalene brachial plexus block to placebo for adjunctive postoperative analgesia after ambulatory shoulder surgery. ${ }^{18}$ We anticipated that mean VAS pain scores on POD 1 after ambulatory hand surgery would be 20 and $40 \mathrm{~mm}$ for the RA and GA groups, respectively, with an SD of $30 \mathrm{~mm}$. To detect a $20 \%$ difference in VAS pain scores on POD 1 with a type I error rate of 0.01 and a power of 0.80 , we calculated that 60 patients (30/group) would be required.

\section{Results}

Preoperatively on the day of surgery, we approached 212 patients in the DSU to recruit and obtain consent for the current study. Eighty-five patients did not meet inclusion criteria for the following reasons: refusal to participate, 69 patients; language barrier, 6; weight $>100$ $\mathrm{kg}, 2$; medication intolerance/allergy, 2; age $<18 \mathrm{yr}, 2$; long-term opioid use, 2 ; psychiatric disease, 1 ; and participation in another clinical trial, 1. Twenty-seven patients gave consent and were randomized but were subsequently excluded for the following reasons: anesthesiologist favored RA, 11 patients; lost to followup, 9; full stomach, 3; surgery canceled, 2; withdrawal of consent, 1; and intraarticular steroid injection during surgery, 1. A total of 100 patients complied with the protocol and completed the study.

Patient characteristics (except weight), medical history, American Society of Anesthesiologists physical status, preoperative VAS and PDI scores, surgeon, and types of hand surgery did not differ between the RA and GA groups (table 1). Procedure types included tendon and nerve repair, fracture fixation and arthrodesis, digital amputation, and hardware removal.

There was no difference in intraoperative tourniquet pressure (RA, $245.0 \pm 35.3 \mathrm{mmHg}$; GA, $245.0 \pm$ $35.3 \mathrm{mmHg} ; P=1.000)$ or duration of tourniquet inflation $(\mathrm{RA}, 44.6 \pm 20.3 \mathrm{~min} ; \mathrm{GA}, 51.6 \pm 22.4 \mathrm{~min} ; P=$ $0.103)$ between groups. During RA administration, the median amount of needle-skin punctures (i.e., attempts) was $1 /$ patient (range, 1-7). Needle-skin penetration lasted $7.7 \pm 5.8 \mathrm{~min} /$ patient. Although transient paresthesiae occurred in 33 patients, no patients reported intense pain on injection of local anesthetic suggestive of intraneural injection. Inadequate RA was recorded in 14 patients, of whom all required intraoperative local anesthetic infiltration by the surgeon (mean dose of lidocaine in 5 patients, $77.1 \pm 26.9 \mathrm{mg}$; mean dose of bupivacaine in 9 patients, $35.0 \pm 26.9 \mathrm{mg}), 11$ required intraoperative fentanyl supplementation (mean dose, $59.1 \pm$ $16.9 \mu \mathrm{g}$ ), and 7 required intraoperative propofol (mean dose, $144.3 \pm 137.8 \mathrm{mg}$ ). Five patients (10\%) randomized to RA required conversion to GA because of block failure. 
Table 1. Patient Characteristics

\begin{tabular}{|c|c|c|c|}
\hline & RA Group $(n=50)$ & GA Group $(n=50)$ & Significance, $P$ Value \\
\hline Age, $\mathrm{yr}^{\star}$ & $42.5(16)$ & 44.7 (14.3) & 0.48 \\
\hline Sex, M/F & $28 / 22$ & $34 / 16$ & 0.2 \\
\hline Weight, kg & $74.3(15.7)$ & $80.8(16.9)$ & 0.048 \\
\hline ASA, I/II/III & $22 / 26 / 2$ & $19 / 28 / 3$ & 0.8 \\
\hline Preoperative VAS* & $23.4(27.4)$ & $33(29.9)$ & 0.1 \\
\hline Preoperative PDI† & $27.5(0-51)$ & $32(0-57)$ & 0.3 \\
\hline Surgical duration, $\min ^{*}$ & $54.4(23.1)$ & $62.0(24.4)$ & 0.1 \\
\hline Tourniquet time, $\min ^{\star}$ & 44.6 (20.3) & $51.6(22.4)$ & 0.1 \\
\hline Tourniquet pressure, $\mathrm{mmHg}^{*}$ & 245 (35.3) & 245 (35.3) & 1.0 \\
\hline
\end{tabular}

* Data presented as mean (SD). † Data presented as median (range).

$\mathrm{ASA}=$ American Society of Anesthesiologists (physical status); GA = general anesthesia; PDI = Pain-Disability Index; RA = regional anesthesia; VAS = visual analog scale.

Of the 50 patients randomized to GA, 44 patients were treated with a laryngeal mask airway, whereas 6 required tracheal intubation for airway management.

The anesthesia time (time from which the anesthesiologist attended the patient to time at which patient care was transferred in PACU) was shorter in the GA group (RA, $112.3 \pm 27.3 \mathrm{~min}$; GA, $92.6 \pm 25.9 \mathrm{~min} ; P<0.001$ ) primarily because in the RA group this included the time spent instituting RA in the regional block room before entering the OR. The duration of surgical time was similar between groups (RA, $54.4 \pm 23.1 \mathrm{~min}$; GA, $62.0 \pm$ $24.4 \mathrm{~min} ; P=0.111$ ); however, the total (surgical plus anesthesia) time in the OR was significantly shorter in the RA group (RA, $71.3 \pm 22.9 \mathrm{~min}$; GA, $90.0 \pm 25.7$ min; $P<0.001)$. Upper airway obstruction developed in one patient in the GA group after extubation of the airway, and the patient was admitted to hospital overnight for observation.

After surgery, more RA patients (RA, 49; GA, 27; $P<$ 0.001 ) were fast-track eligible, and PACU recovery time was shorter in the RA group (RA, $34.5 \pm 22.7 \mathrm{~min}$; GA, $73.2 \pm 31.0 \mathrm{~min} ; P<0.001)$. Although there were no differences in DSU time between groups (RA, $65.9 \pm 43$ min; GA, $68.7 \pm 33.4 \mathrm{~min} ; P=0.7$ ) if the time spent both in PACU and DSU are combined the RA patients spent significantly less time in the hospital before dis- charge after leaving the OR (RA, $100.4 \pm 45.6 \mathrm{~min}$; GA, $142.6 \pm 49 \mathrm{~min} ; P<0.001)$.

In addition, more RA patients achieved home-readiness criteria at $30 \mathrm{~min}(\mathrm{RA}, 49 ; \mathrm{GA}, 36 ; P<0.01)$ and $90 \mathrm{~min}$ (RA, 50; GA, 44; $P<0.05$ ) postoperatively, but there was no statistical difference at $60 \mathrm{~min}$ (RA, 49; GA, 43; $P=0.12$ ) or $120 \mathrm{~min}$ (RA, 50; GA, 46; $P=0.16$ ).

During in-hospital recovery, the time to first analgesic requirement was longer in the RA group (RA, $97.6 \pm$ $50.2 \mathrm{~min} ; \mathrm{GA}, 29.9 \pm 22.8 \mathrm{~min} ; P<0.001)$. Fentanyl consumption (RA, $7.0 \pm 22.0 \mu \mathrm{g} ; \mathrm{GA}, 77.5 \pm 50.3 \mu \mathrm{g}$; $P<0.001$ ), oral morphine equivalent consumption (RA, $7.3 \pm 15.2 \mathrm{mg} ; \mathrm{GA}, 22.8 \pm 18.1 \mathrm{mg} ; P<0.01$ ) (table 2 ), and VAS pain scores (fig. 1) were lower in the RA group. A greater number of GA patients (GA, 12; RA, 3; $P<$ 0.05) experienced nausea, vomiting, or both requiring antiemetics during recovery in the hospital. Two of the three RA patients who required antiemetics for nausea, vomiting, or both in the hospital had undergone conversion to GA for a failed brachial plexus block.

On PODs 1, 7, and 14, there were no differences in pain (fig. 1) or satisfaction scores or in daily or cumulative oral morphine equivalent consumption (table 2). All patients reported moderate pain on PODs 1 and 7 (fig. 1). The incidence of nausea or vomiting did not differ between groups on PODs 1, 7, and 14. There was no

Table 2. Postoperative Analgesic Consumption and Adverse Effects

\begin{tabular}{|c|c|c|c|}
\hline & RA Group $(n=50)$ & GA Group $(n=50)$ & Significance, $P$ Value \\
\hline Time to first analgesic request, $\min ^{*}$ & $97.6(50.2)$ & $29.9(22.8)$ & $<0.001$ \\
\hline Intravenous fentanyl in PACU, $\mu \mathrm{g}^{*}$ & $7(22)$ & $77.5(50.3)$ & $<0.001$ \\
\hline $\begin{array}{l}\text { Oral morphine equivalent in PACU } \\
\text { and DSU, } \mathrm{mg}^{\star}\end{array}$ & 7.3 (15.2) & $22.8(18.1)$ & $<0.01$ \\
\hline $\begin{array}{l}\text { Nausea and vomiting in PACU/DSU, } \\
\text { No. of patients }\end{array}$ & $3+$ & 12 & $<0.05$ \\
\hline $\begin{array}{l}\text { Analgesic consumption POD 1, mg } \\
\text { oral morphine* }\end{array}$ & $42.1(35.1)$ & $53.3(47.9)$ & 0.2 \\
\hline $\begin{array}{l}\text { Analgesic consumption POD } 7, \mathrm{mg} \\
\text { oral morphine* }\end{array}$ & $20.6(31.4)$ & $21.5(43.3)$ & 0.9 \\
\hline $\begin{array}{l}\text { Analgesic consumption POD 14, mg } \\
\text { oral morphine* }\end{array}$ & $13.2(22.3)$ & $12.3(26.5)$ & 0.9 \\
\hline
\end{tabular}

* Data presented as mean (SD). † Two patients received general anesthesia (GA) for regional block failure.

$\mathrm{DSU}=$ day surgery unit; PACU = postanesthesia care unit; POD = postoperative day; $\mathrm{RA}=$ regional anesthesia. 


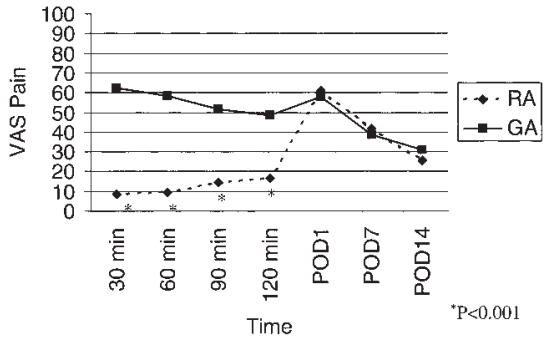

Fig. 1. Visual analog scale (VAS) scores in the hospital and at home up to postoperative day (POD) 14. VAS rating of pain intensity ( $y$-axis) versus time $(\mathbf{x}$-axis). $\mathbf{G A}=$ general anesthesia; $\mathbf{R A}=$ regional anesthesia.

difference in PDI scores on POD 14. Both groups reported equally high satisfaction scores on PODs 1,7 , and 14 regarding the type of anesthetic they received for their hand surgery.

The incidence of postoperative paresthesiae in RA patients at 2 weeks postoperatively was not associated with the number of needle-skin punctures $(P=0.875)$, duration of needle-skin penetration $(P=0.922)$, or occurrence of transient paresthesiae $(P=0.465)$ on RA administration. The incidence of reported paresthesiae was similar in both groups at 2 weeks (GA, 22; RA, 20; $P=0.44)$.

\section{Discussion}

This is the first prospective randomized controlled study comparing RA against GA for hand surgery. We did not confirm our hypothesis that the improved early pain control with RA would confer analgesic benefits up to 14 days after surgery. In fact, both groups had moderate pain both at $24 \mathrm{~h}$ and 7 days after surgery, indicating that patients have significant pain at home after ambulatory hand surgery. The failure to demonstrate a preventive analgesic benefit ${ }^{5}$ is probably because central sensitization occurred in both groups after the anesthetic effect had receded, especially in the first few days after surgery, when moderate pain was experienced. This may have been different had we used a long-acting local anesthetic or local anesthetic infusion to prolong pain control into POD 1. However, recent studies indicate that the use of local anesthetics alone to produce preventive analgesia has disappointing results. ${ }^{19}$ Although both groups did receive ketorolac before surgery, greater efforts to reduce central sensitization, such as the use of an $N$-methyl-D-aspartate receptor antagonist, ${ }^{20}$ may have been more successful in reducing pain in the days and weeks after surgery.

Despite our disappointing results with regard to pain control after discharge, we have demonstrated that brachial plexus block provides a significantly better quality of recovery in comparison to GA. Patients who underwent RA benefited from superior postoperative pain control with decreased adverse effects that facilitated fast-track eligibility and hospital discharge after ambulatory hand surgery compared with GA.

In contrast to existing retrospective data supporting improved satisfaction rates in patients who receive RA compared with GA for ambulatory upper extremity surgery, ${ }^{2}$ our results suggest equally high satisfaction scores for patients in both groups. The high satisfaction scores observed in the GA group may well reflect the minimal side effect profile of the general anesthetic agents used in the current study. However, patient satisfaction with anesthesia may be influenced by many other factors that occur in the perioperative period. ${ }^{21}$

By decreasing the duration of time the patient spends in the OR and the PACU, we can reduce perioperative costs. ${ }^{1}$ The current study demonstrates that RA significantly shortens time in both the OR and the PACU compared with GA. The most likely reason for the shortened total intraoperative time observed in the RA group is that brachial plexus blockade was performed outside the OR in a separate regional block room. Longer anesthesia times were observed in RA patients, but the costs of valuable OR time were spared and efficiency was maximized because the patients had an anesthetized limb and were ready for surgery as soon as they were transferred to the OR. This time efficiency could, at best, lead to the ability to perform more cases per day or at least avoid the cancellation of cases due to delay in the OR. However, the use of a separate block room has cost implications in itself that may outweigh any benefit gained from the reduced OR time. ${ }^{22}$

We demonstrated that with RA, patients spent significantly less time in the hospital and were discharged significantly earlier after surgery then with GA $(P<$ $0.001)$, primarily because of a reduction in PACU time. Patients in the GA group had greater pain, required significantly more analgesics while in the hospital, and experienced greater nausea. However, there was actually no difference between groups in terms of time spent in the DSU, despite the overall reduced time spent in the hospital after surgery in the RA group. Time spent in the DSU is affected by numerous confounding factors, such as dressing the patient and the availability of patient chaperones and transport, ${ }^{7}$ and this may have resulted in our inability to demonstrate a reduction in DSU time in the RA group.

The most recent American Society of Anesthesiologists Closed Claims database analysis in 1999 revealed that the use of RA is more frequently associated with claims involving nerve damage compared with $\mathrm{GA}^{23}$ but the mechanism of nerve injury has yet to be defined. RA may cause nerve injury by way of direct needle trauma, local anesthetic toxicity, or pressure-induced ischemia. Alternatively, nerve injury may be unrelated to RA and due to patient position, prolonged tourniquet inflation, or surgical technique. Nevertheless, many anesthesiologists do not provide RA for fear of medicolegal action stemming 
from nerve damage. ${ }^{24}$ Despite widely held beliefs that RA causes nerve damage more readily than GA, there have been no randomized trials comparing adverse postoperative neurologic outcome in patients receiving RA versus GA for upper extremity surgery. The current study demonstrates that, although the incidence of postoperative paresthesiae is surprisingly high in all patients after ambulatory hand surgery, there is no difference in neurologic symptoms between RA and GA groups up to 2 weeks after surgery. These data tell us nothing about the cause of the neurologic symptoms, but they do tell us that neurologic symptoms are common after hand surgery and that all potential causes of such symptoms should be explored before apportioning blame. ${ }^{25}$

This study has a number of limitations, including use of an open-label technique; a short-acting local anesthetic agent; and a single-shot, single-endpoint brachial plexus block technique.

Although all data in the hospital were collected by an unblinded research assistant, it would have been extremely difficult to blind a research assistant to a patient recovering from RA compared with GA. However, the validated scoring systems used for data collection in the hospital (such as Aldrete and Post-Anesthesia Discharge Scoring System) may reduce the potential for introduction of bias. All data recorded at home were by patient self-report in a diary, without contact with any member of the research team. Telephone calls were made to remind patients to complete the diary, but no assistance was given with completion.

Although single-endpoint axillary block techniques are associated with a lower success rate then multiple-endpoint techniques, ${ }^{26}$ we wanted to use a technique that is commonly used and therefore able to be generalized to a wider population. Certainly the block failure rate observed in this study could be improved with the use of a multiple-endpoint technique. However, transarterial axillary brachial plexus blockade has an easily recognizable endpoint and is easy to teach and learn. For this reason, it is the preferred axillary technique taught at our institution for ambulatory hand surgery given the record of success and safety with which it is associated. ${ }^{27}$

Lidocaine, $1.5 \%(10 \mathrm{mg} / \mathrm{kg})$, is our local anesthetic agent of choice for axillary brachial plexus blockade in patients undergoing ambulatory hand surgery because of its rapid onset, but it is limited by a short duration of action. The use of a longer-acting agent may have extended duration of analgesia into the first POD.

\section{Future Directions}

Efforts should be directed at reducing the pain experienced by patients in the early postoperative period at home after ambulatory hand surgery. Improved patient education could provide a simple but beneficial intervention. Prolongation of analgesia with multimodal techniques ${ }^{28}$ such as the use of controlled-release opioids, ${ }^{29}$ nonsteroidal antiinflammatory agents, or both could provide benefit. Recently evaluated techniques such as patient-controlled RA that enable continuous axillary brachial plexus blockade at home ${ }^{30}$ may help to reduce the moderate pain that patients experience during the first week after ambulatory hand surgery.

\section{Summary}

In conclusion, RA when compared with GA did not result in better pain control at home up to 14 days following ambulatory hand surgery. However RA did provide improved early pain control with less adverse effects and faster hospital discharge.

\section{References}

1. Chan VW, Peng PW, Kaszas Z, Middleton WJ, Muni R, Anastakis DG, Graham BA: A comparative study of general anesthesia, intravenous regional anesthesia, and axillary block for outpatient hand surgery: Clinical outcome and cost analysis. Anesth Analg 2001; 93:1181-4

2. Brown AR, Weiss R, Greenberg C, Flatow EL, Bigliani LU: Interscalene block for shoulder arthroscopy: Comparison with general anesthesia. Arthroscopy 1993; 9:295-300

3. D'Alessio JG, Rosenblum M, Shea KP, Freitas DG: A retrospective comparison of interscalene block and general anesthesia for ambulatory surgery shoulder arthroscopy. Reg Anesth 1995; 20:62-8

4. Prabhu A, Chung F: Anaesthetic strategies towards developments in day care surgery. Eur J Anaesthesiol Suppl 2001; 23:36-42

5. Kissin I: Pre-emptive analgesia: Terminology and clinical relevance. Anesth Analg 1994; 79:809-10

6. Woolf CJ, Chong MS: Preemptive analgesia: Treating postoperative pain by preventing the establishment of central sensitization. Anesth Analg 1993; 77: 362-79

7. Pavlin DJ, Rapp SE, Polissar NL, Malmgren JA, Koerschgen M, Keyes H: Factors affecting discharge time in adult outpatients. Anesth Analg 1998; 87: 816-26

8. Epple J, Kubitz J, Schmidt H, Motsch J, Bottiger BW, Martin E, Bach A: Comparative analysis of costs of total intravenous anaesthesia with propofol and remifentanil vs. balanced anaesthesia with isoflurane and fentanyl. Eur J Anaesthesiol 2001; 18:20-8

9. Smith MP, Sprung J, Zura A, Mascha E, Tetzlaff JE: A survey of exposure to regional anesthesia techniques in American anesthesia residency training programs. Reg Anesth Pain Med 1999; 24:11-6

10. van Vlymen JM, White PF: Outpatient anesthesia, Anesthesia, 5th edition. Edited by Miller RD. Philadelphia, Churchill Livingstone, 2000, pp 2213-40

11. Bruyns CN, Jaquet JB, Schreuders TA, Kalmijn S, Kuypers PD, Hovius SE: Predictors for return to work in patients with median and ulnar nerve injuries. J Hand Surg [Am] 2000; 28:28-34

12. Singelyn FJ, Deyaert M, Joris D, Pendeville E, Gouverneur JM: Effects of intravenous patient-controlled analgesia with morphine, continuous epidural analgesia, and continuous three-in-one block on postoperative pain and knee rehabilitation after unilateral total knee arthroplasty. Anesth Analg 1998; 87: $88-92$

13. Tait RC, Pollard CA, Margolis RB, Duckro PN, Krause SJ: The Pain Disability Index: Psychometric and validity data. Arch Phys Med Rehabil 1987; 68:438-41

14. Aldrete JA: Modifications to the postanesthesia score for use in ambulatory surgery. J Perianesth Nurs 1998; 13:148-55

15. White PF, Song D: New criteria for fast-tracking after outpatient anesthesia: A comparison with the modified Aldrete's scoring system. Anesth Analg 1999; 88:1069-72

16. Chung F, Chan VW, Ong D: A post-anesthetic discharge scoring system for home readiness after ambulatory surgery. J Clin Anesth 1995; 7:500-6

17. Canadian Pharmacists Association: Compendium of Pharmaceuticals and Specialties, 39th edition. Ottawa, Canadian Pharmacists Association, 2004

18. Al Kaisy A, McGuire G, Chan VW, Bruin G, Peng P, Miniaci A, Perlas A: Analgesic effect of interscalene block using low-dose bupivacaine for outpatient arthroscopic shoulder surgery. Reg Anesth Pain Med 1998; 23:469-73

19. Katz J, McCartney CJL: Update on pre-emptive analgesia. Curr Opin Anesthesiol 2002; 15:435-41

20. McCartney CJL, Sinha A, Katz J: A qualitative systematic review of the role of N-methyl-D-aspartate receptor antagonists in preventive analgesia. Anesth Analg 2004; 98:1385-400 
21. Wu CL, Naqibuddin M, Fleisher LA: Measurement of patient satisfaction as an outcome of regional anesthesia and analgesia: A systematic review. Reg Anesth Pain Med 2001; 26:196-208

22. Armstrong KP, Cherry RA: Brachial plexus anesthesia compared to general anesthesia when a block room is available. Can J Anaesth 2004; 51:41-4

23. Cheney FW, Domino KB, Caplan RA, Posner KL: Nerve injury associated with anesthesia: A closed claims analysis. ANesthesiology 1999; 90:1062-9

24. Katz J: A survey of anesthetic choice among anesthesiologists. Anesth Analg 1973; 52:373-5

25. Jankowski CJ, Keegan MT, Bolton CF, Harrison BA: Neuropathy following axillary brachial plexus block: Is it the tourniquet? ANESTHesiology 2003; 99: $1230-2$
26. Coventry DM, Barker KF, Thomson M: Comparison of two neurostimulation techniques for axillary brachial plexus blockade. Br J Anaesth 2001; 86:80 -3 27. Urban MK, Urquhart B: Evaluation of brachial plexus anesthesia for upper extremity surgery. Reg Anesth 1994; 19:175-82

28. Jin F, Chung F: Multimodal analgesia for postoperative pain control. J Clin Anesth 2001; 13:524-39

29. Reuben SS, Connelly NR, Maciolek H: Postoperative analgesia with controlled-release oxycodone for outpatient anterior cruciate ligament surgery Anesth Analg 1999; 88:1286-91

30. Ilfeld BM, Morey TE, Enneking FK: Continuous infraclavicular brachial plexus block for postoperative pain control at home: A randomized, doubleblinded, placebo-controlled study. ANESTHESIOLOGY 2002; 96:1297-304 